\title{
Rare Contralateral Ocular Complication after Intraoral Anesthetic Injection: A Case Series
}

\author{
Soumya $\mathrm{S}^{1}$, Faisal MA Gaffoor ${ }^{2}$, Rethi Gopakumar ${ }^{3}$, Sabari Girish $C^{4}$
}

\begin{abstract}
Intraoral administration of local anesthesia is inevitable for most of the procedures in endodontics. It may be accompanied by ocular complications that are reasonably rare and transient in nature but can cause discomfort for both the patient and the clinician. Most literatures have reported complications that occurred ipsilateral to the injection site. Very rarely contralateral disturbances of eyes are reported. This case series reports two cases in which one case was followed by ptosis, pain, and discomfort of an eye after administering an inferior alveolar nerve block. The other was immediately followed by twitching, pain, and discomfort of an eye after the maxillary infiltration technique. Complete recovery was achieved without any interventions. In this article, we are reporting multiple cases of contralateral complications along with a scientific explanation for this phenomenon and its prevention and management.

Key messages: Although contralateral ocular complications are transient, potential sequelae including blindness should be considered. Measures like initial aspiration, slow injection of anesthetic solution, usage of a $25-\mathrm{G}$ needle, and management of patient anxiety reduce the chance of ocular complications.

Keywords: Case series, Complication, Contralateral, Local anesthesia, Ocular.

Conservative Dentistry and Endodontic Journal (2021): 10.5005/jp-journals-10048-0071
\end{abstract}

\section{INTRODUCTION}

Millions of local anesthetic cartridges are administered in dentistry each year, yet ocular complications appear to be rarely reported in the literature. Ocular complications range from transient unilateral vertical diplopia reported by Chisci et al. ${ }^{1}$ to the most calamitous ocular outcome reported in 1957 by Walsh, ${ }^{2}$ where an intraoral anesthetic injection of $2 \%$ procaine-adrenaline $(1: 50,000)$ solution resulted in permanent blindness. Commonly reported responses are seen on the ipsilateral side of the injection site. Mentioned below are cases that presented with contralateral ocular changes after routine inferior alveolar nerve block (IANB) and maxillary buccal infiltration technique.

\section{Case Descriptions}

\section{Case 1}

A female patient with an age of 40 years reported to the department with pain in relation to the left upper posterior region for the past 2 days. The pain was severe in nature and aggravated during the night. It was her first dental visit and she had no relevant medical history. On intraoral examination, a large cavitated lesion with involvement of pulp was observed in relation to the left maxillary first molar. Radiographic examination revealed the pulpal involvement. The vitality test was positive. The tooth was diagnosed as symptomatic irreversible pulpitis on the left maxillary first molar whose root canal treatment $(\mathrm{RCT})$ was planned under local anesthesia.

Before administering anesthesia, evaluation of the vital signs was done and no deviation from normal observed. Sensitivity test to local anesthesia revealed no allergic response. A long (30 mm) 28-G needle was used to give routine buccal infiltration anesthesia. During the injection, multiple aspirations were done. $0.5 \mathrm{~mL}$ of the $2 \%$ lidocaine (1:80,000 epinephrine) was injected slowly. Immediately after injection, the patient complained of discomfort

\begin{abstract}
${ }^{1-4}$ Department of Conservative Dentistry and Endodontics, Noorul Islam College of Dental Sciences, Thiruvananthapuram, Kerala, India

Corresponding Author: Soumya S, Department of Conservative Dentistry and Endodontics, Noorul Islam College of Dental Sciences, Thiruvananthapuram, Kerala, India, Phone: +91 9496351208, e-mail: sspbloo9@gmail.com

How to cite this article: Soumya S, Gaffoor FMA, Gopakumar R, et al. Rare Contralateral Ocular Complication after Intraoral Anesthetic Injection: A Case Series. Cons Dent Endod J 2021;6(1):22-25.
\end{abstract}

Source of support: Nil

Conflict of interest: None

in the contralateral eye. We stopped all the procedures, reassured the patient, and were brought to the supine position. She reported involuntary movement of eyelids and twitching which increased with time. The patient also complained of pain and discomfort in relation to the contralateral eye. On inquiry, she gave no history of such experience. Physical examination was done which revealed normal vital signs and absence of unconsciousness. Her mental status was alert, although she appeared slightly nervous. The patient was subjected to an ocular test which revealed normal eye movements in all directions. The vision was checked by making the patient read with both eyes separately and the vision was found to be normal.

Symptoms decreased after 30 minutes but did not subside completely. Any problem with her eyesight was ruled out. The patient was re-examined the next day and on review, eye movements were found to be normal and she reported complete recovery about 6 hours after the first episode. Root canal treatment was initiated and anesthetic injection was successfully administered without any complications. The symptoms did not recur during her subsequent visits either. 


\section{Case 2}

A 53-year-old female patient had reported a chief complaint of pain in relation to the right lower back teeth region for 1 week. The pain was severe and occurred at intervals, aggravating during the course of the night. The patient had no relevant medical history. Root canal treatment of the left mandibular first molar followed by a full metal crown was recorded as the past dental history. Intraoral examination revealed a cavitated lesion with involvement of pulp on the occlusal surface of the tooth. On radiographic examination, pulpal involvement of carious lesion and widening of the periodontal ligament space around both the roots were observed. There was a delayed response to vitality tests and was sensitive to vertical percussion too. The diagnosis was symptomatic irreversible pulpitis with symptomatic apical periodontitis in relation to the right mandibular first molar and RCT was the preferred treatment plan.

Before applying anesthesia, the patient's vital signs were confirmed to be within normal levels. Sensitivity test to local anesthesia revealed no allergic response. A long $(30 \mathrm{~mm}) 28-\mathrm{G}$ needle was used for a routine IANB anesthesia. Multiple aspirations were performed during the injection which revealed no blood. 1.8 $\mathrm{mL}$ of $2 \%$ lidocaine (containing 1:100,000 epinephrine) was injected slowly. Immediately after injection, the patient complained of pain and discomfort that had not been experienced earlier. The patient experienced an inability to open the eye to a normal extent suggestive of ptosis on the contralateral side. Immediately, all the dental procedures were stopped and the patient was brought to a supine position.

The vital signs of the patient were normal and the patient was conscious and mentally alert. An ocular test was done where the patient was asked to move the eye in all directions. The patient experienced moderate difficulty in moving upward and downward. A vision test was performed for both eyes and it was found to be normal.

All symptoms disappeared in about 1 hour after the initial observation. Root canal treatment was completed on the same day under local anesthesia. The 3-day follow-up of the patient revealed nothing conspicuous after leaving the clinic. The symptoms did not recur during subsequent visits.

\section{Discussion}

Painless endodontic treatment is a result of an accurate injection technique given in an appropriate injection site using an effective local anesthetic agent. ${ }^{3}$

For procedures on the maxilla, quite often, infiltration anesthesia will be sufficient due to the thin and porous nature of the cortical plate. For mandibular procedures, nerve-block anesthesia of the inferior alveolar, lingual, and buccal nerves is often required. ${ }^{4}$

Inferior alveolar nerve anesthesia (45.8\%) or posterior superior alveolar nerve anesthesia is the anesthetic technique that commonly results in ocular complications. According to the literatures, the most common ocular complication is diplopia (39.8\%) followed by ptosis (16.7\%), mydriasis (14.8\%), and amaurosis $(13.0 \%)^{5}$

Although there have been multiple reports of ipsilateral ocular complications after administration of anesthesia, cases of contralateral ocular changes after maxillary and mandibular anesthetic technique were unusual observations. This demanded, attending to etiology and possible complications.

As there is no literature support for contralateral ocular changes after maxillary buccal infiltration technique, here we are considering various hypotheses which might explain the etiology.

Complications occurring away from the site of injection of anesthesia can be justified by the accidental entry of a drug into the circulation.

Accidental deposition of anesthetic solution through the extraosseous branch of posterior superior alveolar artery (the third portion of the maxillary artery supplying maxillary molar and maxillary sinus) will then lead to infraorbital artery which supplies the lower eyelid, cheek, and lateral nose. ${ }^{4}$ The infraorbital artery communicates with the facial artery which in turn joins the ophthalmic artery near the medial canthus of the eye.

The anesthetic solution may get diffused to areas in and around the maxillary artery. Through the middle meningeal artery, one of the main branches of the maxillary artery, it then reaches the cerebral region. However, in $7.1 \%$ of cases, the middle meningeal artery gives rise to the ophthalmic artery. ${ }^{6}$ Thus, the anesthetic solution enters the ophthalmic artery from the middle meningeal artery. The ophthalmic artery supplies orbital structures including the extraocular muscles which result in widespread ocular symptoms (Fig. 1A). ${ }^{7}$

The anastomosis across the median plane may facilitate the spread of anesthetic solution to the contralateral side resulting in the contralateral changes of the eye (Fig. 2).

Regarding the venous pathway, usage of a small-gauge needle may cause accidental entry into the dense venous pterygoid plexus which enters the ophthalmic vein via retrograde flow and end up into the cavernous sinus. Also, the extrinsic muscles of the eye are directly connected with the ophthalmic vein via the inferior orbital fissure (IOF). ${ }^{1}$ The two cavernous sinuses also receive the superior
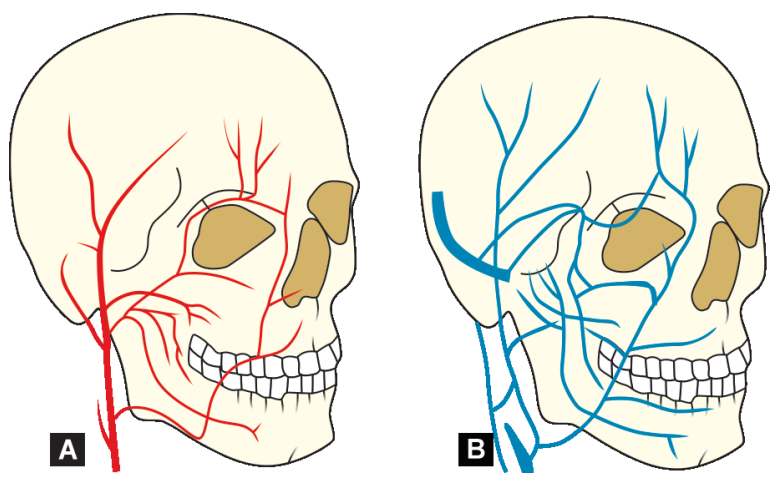

Figs $1 \mathrm{~A}$ and B: (A) Diagram demonstrates arterial anastomosis on the ipsilateral side of the face. (1) Common carotid artery. (2) External carotid artery. (3) Internal carotid artery. (4) Facial artery. (5) Maxillary artery. (6) Inferior alveolar artery. (7) Mental artery. (8) Buccal artery. (9) Infraorbital artery. (10) Ophthalmic artery. (11) Supraorbital artery. (12) Supratrochlear artery. (13) Dorsal nasal artery. Arterial anastomoses occur between maxillary, buccal, infraorbital, inferior alveolar, and mental arteries (branches of external carotid artery). Facial artery anastomoses with supraorbital, supratrochlear, and dorsal nasal arteries; (B) Diagram demonstrates venous anastomosis on the ipsilateral side of face. (1) Angular vein. (2) Superior ophthalmic vein. (3) Inferior ophthalmic vein (4) Facial vein. (5) Deep facial vein. (6) Cavernous sinus. (7) Pterygoid venous plexus. (8) Inferior alveolar vein 


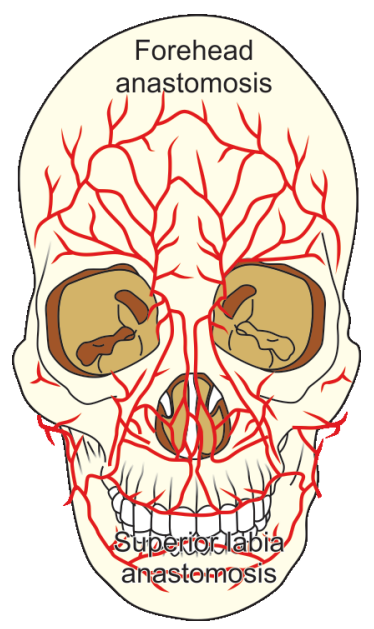

Fig. 2: Diagram shows arterial anastomosis across the median plane of the face

ophthalmic veins and communicate across the median plane via the intercavernous venous channels (Fig. 1B).

The twitching of the eye manifested by the contralateral side could be due to the effect of anesthetic solution reaching the contralateral local tissues surrounding the facial muscles through the arterial and venous anastomosis. Manifestations even at low concentration can be justified by the thinner perineurial and connective tissue sheaths of the fine terminal branches of the facial nerve. ${ }^{8}$

Another possible scenario is the central effect of the anesthetic solution on the ipsilateral motor cortex manifesting as contralateral muscle dysfunction. Reverse flow from the external to the internal carotid artery results in diffusion of the solution to the middle cerebral artery which in turn supplies the motor cortex for the head region. The effect of the local anesthetic on the ipsilateral motor cortex may adversely affect the contralateral cranial nerve function. ${ }^{9}$

The possible cause for contralateral ocular manifestations following IANB was described by Kempster et al. ${ }^{8}$ Suggested hypothesis includes arterial and venous anastomosis ipsilaterally and along median planes, due to central effect on the motor cortex. The symptoms may also be anxiety-related.

Thus, in the second case, the probable hypothesis could be accidental diffusion of anesthetic solution into the circulation. Intra-arterial injection into an inferior alveolar artery (nerve located anterior to vessels) is followed by the retrograde flow into the maxillary artery and then to the middle meningeal artery. Although aspiration is negative, a slight movement of the patient or syringe may cause penetration of the arterial wall. ${ }^{3,10}$ Inadvertent administration of local anesthetic solution into an inferior alveolar vein can cause entry of solution to a facial vein and ophthalmic vein which communicate with the pterygoid venous plexus. ${ }^{8}$

From the maxillary artery and the pterygoid venous plexus, the anesthetic solution follows the same pathway as explained for maxillary anesthesia to produce contralateral complications.

The IOF communicates with the pterygopalatine fossa which in turn meets the greater palatine canal. Thus, these bony openings facilitate the diffusion of anesthetic solution, especially in patients with unique anatomic variations. ${ }^{1}$

Effect on ipsilateral motor cortex can also be considered.
However, it is possible that the symptoms were idiosyncratic or related to a sympathetic emotional response from the patient.

Thus, ocular complications might be due to any of these reasons.

To prevent iatrogenic anesthetic complications, the precautions to be taken include,

- Look for swelling or blanching of the site which indicates extravascular injection.

- Aspiration must be done on at least two planes to avoid intravascular injection.

- Deposit the anesthetic solution slowly, emptying a full cartridge in about 60 seconds.

- For injections with less risk of positive aspiration, a 25-G needle is preferred.

- Amount of solution must be in accordance with the guidelines for specific nerve blocks.

- Anatomic landmarks for each injection should be evaluated before giving the nerve block.

- Along with technical considerations, patient management and anxiety reduction should be ensured for successful anesthesia.

The following are the guidelines for better management of the complications that might occur even after utmost care.

- Patient must be reassured. Explain the temporary nature of the complications.

- Use a gauze dressing to cover the affected eye.

- Make sure the patient goes home along with a responsible person.

- If ocular complications persist for $>6$ hours, consultation with an ophthalmologist should be considered. ${ }^{11}$

\section{Conclusion}

Contralateral ocular complications after the administration of local anesthesia are rare but can happen even after proper care and attention. The symptoms may develop from an accidental entry of an anesthetic agent into the arterial or venous circulation either through diffusion into the facial planes or local tissues. Effective management of the patient's anxiety, proper visualization of the anatomical landmarks, multiple aspirations during the injection, and aspiration on at least two planes before injection will help the clinician to reduce the occurrence of ocular complications.

\section{References}

1. Chisci G, Chisci C, Chisci V, et al. Ocular complications after posterior superior alveolar nerve block: a case of trochlear nerve palsy. Int J Oral Maxillofac Surg 2013;42(12):1562-1565. DOI: 10.1016/j. ijom.2013.05.010.

2. Boynes SG, Echeverria Z, Abdulwahab M. Ocular complications associated with local anesthesia administration in dentistry. Dent Clin North Am 2010;54(4):677-686. DOI: 10.1016/j.cden.2010.06.008.

3. Pandey R, Dixit N, Dixit KK, et al. Amaurosis, an unusual complication secondary to inferior alveolar nerve anesthesia: a case report and literature review. J Endod 2018;44(9):1442-1444. DOI: 10.1016/j. joen.2018.05.005.

4. Scarano A, Sinjari B, Lorusso F, et al. Intense, instantaneous, and shooting pain during local anesthesia for implant surgery. J Craniofac Surg 2018;29(8):2287-2290. DOI: 10.1097/SCS.0000000000004575.

5. von Arx T, Lozanoff S, Zinkernagel M. Ophthalmic complications and local anesthesia. Pathophysiology and types of eye complications 
after intraoral dental anesthesia, and clinical recommendations. Swiss Dent J 2014;124(11):1189-1203.

6. Melé MV, Puigdellívol-Sánchez A, Mavar-Haramija M, et al. Review of the main surgical and angiographic-oriented classifications of the course of the internal carotid artery through a novel interactive 3D model. Neurosurg Rev 2020;43(2):473-482. DOI: 10.1007/s10143018-1012-7.

7. Kini YK, Kharkar VR, Kini AY. Transient diplopia with ipsilateral abducent nerve palsy and ptosis following a maxillary local anesthetic injection: a case report and review of literature. Oral Maxillofac Surg 2012;16(4):373-375. DOI: 10.1007/s10006-011-0304-7.
8. Kempster C, Ghabriel M, Kaidonis G, et al. An unusual ocular complication following dental anaesthesia: case report. Aust Dent J 2016;61(3):374-380. DOI: 10.1111/adj.12392.

9. Malamed SF. Handbook of local anesthesia. 6th ed., St Louis: Elsevier; 2013. pp. 311-340.

10. Steenen SA, Dubois L, Saeed $P$, et al. Ophthalmologic complications after intraoral local anesthesia: case report and review of literature. Oral Surg Oral Med Oral Pathol Oral Radiol 2012;113(6):e1-e5. DOI: 10.1016/j.tripleo.2011.07.032.

11. Pragasm M, Managutti A. Diplopia with local anesthesia. Natl J Maxillofac Surg 2011;2(1):82-85. DOI: 10.4103/0975-5950.85861. 